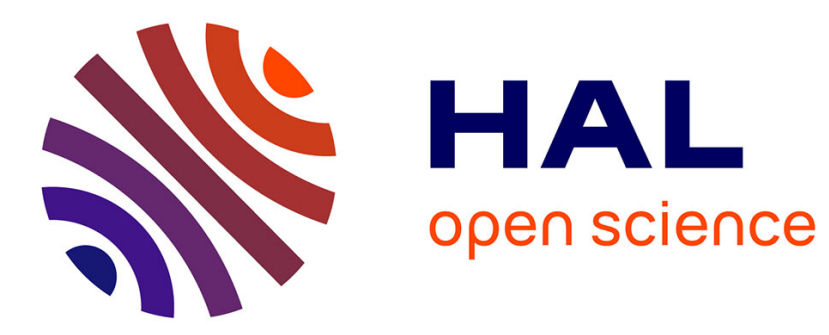

\title{
On the controllability of anomalous diffusions generated by the fractional Laplacian
}

\author{
Luc Miller
}

\section{To cite this version:}

Luc Miller. On the controllability of anomalous diffusions generated by the fractional Laplacian. 2005 . hal-00008809v1

\section{HAL Id: hal-00008809 \\ https://hal.science/hal-00008809v1}

Preprint submitted on 16 Sep 2005 (v1), last revised 1 Mar 2006 (v4)

HAL is a multi-disciplinary open access archive for the deposit and dissemination of scientific research documents, whether they are published or not. The documents may come from teaching and research institutions in France or abroad, or from public or private research centers.
L'archive ouverte pluridisciplinaire HAL, est destinée au dépôt et à la diffusion de documents scientifiques de niveau recherche, publiés ou non, émanant des établissements d'enseignement et de recherche français ou étrangers, des laboratoires publics ou privés. 


\title{
ON THE CONTROLLABILITY OF ANOMALOUS DIFFUSIONS GENERATED BY THE FRACTIONAL LAPLACIAN
}

\author{
LUC MILLER
}

\begin{abstract}
This paper introduces a "spectral observability condition" for a negative self-adjoint operator which is the key to proving the null-controllability of the semigroup that it generates and to estimating the controllability cost over short times. It applies to the interior controllability of diffusions generated by powers greater than $1 / 2$ of the Dirichlet Laplacian on manifolds, generalizing the heat flow. The critical fractional order $1 / 2$ is optimal for a similar boundary controllability problem in dimension one. This is deduced from a subsidiary result of this paper, which draws consequences on the lack of controllability of some one dimensional output systems from Müntz-Szász theorem on the closed span of sets of power functions.
\end{abstract}

In this paper, an observability condition on the spectral subspaces of a negative self-adjoint operator is introduced which ensures fast controllability, i.e. the semigroup generated by this operator is null-controllable in arbitrarily small time. In this asymptotic, it also ensures an upper bound for the controllability cost, i.e. the supremum, over every initial state with norm one, of the norm of the optimal input function which steers it to zero (cf. section 1). This spectral observability condition is the abstract version of a property proved for the Dirichlet Laplacian $\Delta$ on a compact manifold observed on any non empty region in [LZ98, JL99] (cf. [Mil05] for non-compact manifolds).

It applies to the semigroup generated by the fractional Laplacian on manifolds $-(-\Delta)^{\alpha}$ as long as $\alpha>1 / 2$. This semigroup is widely used to describe physical systems exhibiting anomalous diffusions (cf. references in sect.2.1). Thus new interior null-controllability results for such fractional diffusions with non-constant coefficients in any dimension are deduced (the one dimension problem with constant coefficients and one dimensional input was recently considered in [MZ04]). In particular, as the control time $T$ tends to 0 , the controllability cost grows at most like $C_{\beta} \exp \left(c_{\beta} / T^{\beta}\right)$ where $C_{\beta}$ and $c_{\beta}$ are positive constants and $\beta>1 /(2 \alpha-1)$ (n.b. a lower bound of the same form with equality $\beta=1 /(2 \alpha-1)$ holds in the case $\alpha=1$ which corresponds to the heat equation). It is proved in the appendix that a similar one dimensional problem is not controllable from the boundary for $\alpha \in(0,1 / 2]$.

This last result is deduced from a more general remark of independent interest on the lack of controllability of any finite linear combination of eigenfunctions of systems with one dimensional input, based on the generalized Müntz theorem on the completeness of sets of exponentials.

\section{The main Result in the ABstract Setting.}

After recalling the duality between controllability and observability for parabolic semigroups, this section states the main definition and theorem.

1.1. The abstract setting. Let the generator $A$ be a positive self-adjoint operator with domain $D(A)$ on the Hilbert space $\mathcal{H}$ of states which we identify with its dual.

2000 Mathematics Subject Classification. 93B05, 34K35, 58J35. 
The norm in $\mathcal{H}$ is denoted by $\|\cdot\|$ without subscript. Let $\mathcal{H}_{1}$ be the Hilbert space obtained by choosing the graph norm on $D(A)$. Let $\mathcal{H}_{-1}$ be the space dual to $\mathcal{H}_{1}$. We keep the same notation for the extension of $\left\{e^{-t A}\right\}_{t \geqslant 0}$ to a semigroup on $\mathcal{H}_{-1}$.

Let $\mathcal{U}$ be the Hilbert space of inputs which we identify with its dual. Let the observation operator $C$ be a bounded operator from $\mathcal{H}_{1}$ to $\mathcal{U}$ and let the control operator $B \in \mathcal{L}\left(\mathcal{U} ; \mathcal{H}_{-1}\right)$ be its dual. We make the following equivalent admissibility assumptions on these operators (cf. [Wei89]): $\forall T>0, \exists K_{T}>0$,

$$
\begin{gathered}
\forall v_{0} \in D(A), \quad \int_{0}^{T}\left\|C e^{-t A} v_{0}\right\|^{2} d t \leqslant K_{T}\left\|v_{0}\right\|^{2}, \\
\forall u \in L_{\mathrm{loc}}^{2}(\mathbb{R} ; \mathcal{U}), \quad\left\|\int_{0}^{T} e^{-t A} \mathcal{B} u(t) d t\right\|^{2} \leqslant K_{T} \int_{0}^{T}\|u(t)\|^{2} d t .
\end{gathered}
$$

With this assumption, the output map $v_{0} \mapsto C e^{-t A} v_{0}$ from $D(A)$ to $L_{\text {loc }}^{2}(\mathbb{R} ; \mathcal{U})$ has a continuous extension to $\mathcal{H}$. The differential equation:

$$
\dot{\phi}+A \phi=B u, \phi(0)=\phi_{0} \in \mathcal{H}, u \in L_{\text {loc }}^{2}(\mathbb{R} ; \mathcal{U})
$$

has a unique solution $\phi \in C([0, \infty) ; \mathcal{H})$ defined by the integral formula:

$$
\phi(t)=e^{-t A} \phi(0)+\int_{0}^{t} e^{(s-t) A} B u(s) d s .
$$

Definition 1. The parabolic control system (1) is said to be null-controllable in time $T$ if for all $\phi_{0} \in \mathcal{H}$ there is an input function $u \in L_{l o c}^{2}(\mathbb{R} ; \mathcal{U})$ such that the solution $\phi \in C([0, \infty) ; \mathcal{H})$ of (1) satisfies $\phi=0$ at $t=T$.

By duality (cf. [DR77]), it is equivalent to the following observability inequality for solutions $v(t)=e^{-t A} v_{0}$ of the equation without source term: $\dot{v}+A v=0$.

Definition 2. The parabolic semigroup $\left\{e^{-t A}\right\}_{t \geqslant 0}$ is said final-observable through $C$ in time $T$ if there is a positive constant $C_{T}$ such that:

$$
\forall v_{0} \in \mathcal{H}, \quad\left\|e^{-T A} v_{0}\right\| \leqslant C_{T}\left\|C e^{-t A} v_{0}\right\|_{L^{2}(0, T ; \mathcal{U})} .
$$

The best constant $C_{T}$ in (2) can be considered as the controllability cost since it is also the best constant such that, for all initial condition $\phi_{0}$, there is an input $u$ solving the controllability problem in def.1 and satisfying $\|u\|_{L^{2}(0, T ; \mathcal{U})} \leqslant C_{T}\left\|v_{0}\right\|$.

1.2. The main result. Now we introduce the spectral observability condition of order $\gamma>0$ for the generator $A$ and observation operator $C$. This definition is quite natural for dissipative problems as illustrated in sect.3: it allows to compare the free dissipation of high-frequency modes to the cost of controlling low-frequency modes.

Definition 3. Let $\gamma>0$. We say that the observability of $A^{\gamma}$ through $C$ at lowfrequencies with exponential cost holds if there are positive constants $d_{1}$ and $d_{2}$ such that

$$
\forall \mu>1, \forall v \in \mathbf{1}_{A \gamma \leqslant \mu} \mathcal{H}, \quad\|v\| \leqslant d_{2} e^{d_{1} \mu}\|C v\| .
$$

The following main theorem shows that this definition is a relevant condition for estimating how violent fast controls are (this is solved for $\operatorname{dim} \mathcal{H}<\infty$ in [Sei88]).

Theorem 1.1. If def.3 holds with $\gamma \in(0,1)$ then the system (1) is null-controllable in any time $T>0$. Moreover the controllability cost $C_{T}$ (cf. def.2) satisfies

$$
\forall \beta>\frac{\gamma}{1-\gamma}, \exists C_{1}>0, \exists C_{2}>0, \forall T \in(0,1) \quad C_{T} \leqslant C_{2} \exp \left(\frac{C_{1}}{T^{\beta}}\right)
$$




\section{Application to the FRACTiOnal DifFusion}

This section considers the controllability of the semigroup generated by the fractional Laplacian on a manifolds $-(-\Delta)^{\alpha}$, where $\Delta$ denotes the usual Laplacian operator. When the manifold is the whole Euclidean space $R^{d}, \Delta=\frac{\partial^{2}}{\partial x_{1}^{2}}+\cdots+\frac{\partial^{2}}{\partial x_{d}^{2}}$. When the manifold has a boundary, the null Dirichlet condition is always assumed.

2.1. Background on anomalous diffusion models. In recent years, the use of fractional derivatives in dynamical models of physical processes exhibiting anomalously slow or fast diffusion has diffused (cf. the surveys [MK04, SKB02]). Fractional calculus includes various extensions of the usual derivative from integer to real order. In this paper we always use the fractional Laplacian, which is not a local operator when the power $\alpha$ is not an integer. Moreover, the model of anomalous diffusion considered here do not include fractional derivatives, of any kind, with respect to the time variable (cf. [SKB02, GM03, MK04] and references therein).

When the manifold is the whole Euclidean space $R^{d}$, the dynamics considered here is the same as the "isotropic space-fractional diffusion equation" in [Han01], the "strictly space fractional diffusion equation" in [GM03] and the "Lévy fractional diffusion equation" in [MK04]. In this case, the fractional powers of the Laplacian are also known as Riesz fractional derivatives ([GM03]) or Riesz-Weyl operator $([\mathrm{MK} 04])$. They are easily defined through the Fourier transform $\mathcal{F}$ : $\mathcal{F}(-\Delta)^{\alpha} f(\xi)=|\xi|^{2 \alpha} \mathcal{F} f(\xi)$.

The fractional Laplacian $-(-\Delta)^{\alpha}$ with $\alpha \in(0,1]$ generates the rotationally invariant $2 \alpha$-stable Lévy process. For a textbook presentation of this stochastic process, we refer the reader to [Sat99], in particular example 32.7, and for a survey to [App04], in particular example 5 of Lévy process and example 2 of generator. For $\alpha=1$ this process is the Brownian motion $B_{t}$ on $R^{d}$, and for $\alpha<1$ it is subordinated to $B_{t}$ by a strictly $\alpha$-stable subordinator $T_{t}$, so that it writes $B_{T_{t}}$. The convolution kernels of the corresponding semigroups are the rotationally invariant Lévy stable probability distributions, in particular the Gaussian distribution for $\alpha=1$ and the Cauchy distribution for $\alpha=1 / 2$. For $\alpha<1$ these distributions have "heavy tails", i.e. far away they decrease like a power as opposed to the exponential decrease found in the Gaussian, which accounts for the "superdiffusive" behavior of the semigroup. The more restrictive range $\alpha \in(1 / 2,1)$ is the most widely used to model anomalously fast diffusions (cf. [MK04]), and it turns out that the controllability result th.2.1 applies to this range of fractional superdiffusions only. Th.2.1 includes the "subdiffusive" range $\alpha>1$ but it seems that this model has not been considered in the physics literature on anomalously slow diffusion. N.b. the generalized Laplacian operators associated with anisotropic diffusion, also known as the Riesz-Feller derivatives, generate all stable Lévy processes, i.e. including the non invariant ones also called skewed (cf. [Han01], [GM03]). These Lévy processes can be approximated by Lévy flights and references to random walk models of anomalous diffusion can be found in [MK04, GM03].

When the manifold is a domain of the Euclidean space $R^{d}$, the Markov process generated by the fractional Dirichlet Laplacian $-(-\Delta)^{\alpha}$ with $\alpha \in(0,1]$ can be obtained by killing the Brownian motion on $R^{d}$ upon exiting the domain then subordinating the killed Brownian motion by the subordinator $T_{t}$ introduced above (cf. [SV03]). N.b. reversing the order of killing and subordination yields another process which seems to have been investigated earlier and further.

2.2. Controllability of some fractional diffusions. Let $M$ be a smooth connected complete $n$-dimensional Riemannian manifold with metric $g$ and boundary $\partial M$. When $\partial M \neq \emptyset, M$ denotes the interior and $\bar{M}=M \cup \partial M$. Let $\Delta$ denote the Dirichlet Laplacian on $L^{2}(M)$ corresponding to $g$ with domain $D(\Delta)=$ 
$H_{0}^{1}(M) \cap H^{2}(M)$ (n.b. it denotes a negative operator). Let $T$ be a positive time and let $\chi_{\Omega}$ denote the characteristic function of an open subset $\Omega \neq \emptyset$ of $\bar{M}$.

If $M$ is not compact, assume that $\Omega$ is the exterior of a compact set $K$ such that $K \cap \bar{\Omega} \cap \partial M=\emptyset$. In this setting, the observability of $(-\Delta)^{1 / 2}$ through $C$ at low-frequencies with exponential cost holds (cf. def.3). When $M$ is compact this is an inequality on sums of eigenfunctions proved as th.3 in [LZ98] and th.14.6 in [JL99]. This was generalized to non compact $M$ in [Mil05]. Applying th.1.1, with $\mathcal{H}=\mathcal{U}=L^{2}(M), A=(-\Delta)^{\alpha}$ and $B=C \in \mathcal{L}(\mathcal{H} ; \mathcal{U})$ equal to the multiplication by $\chi_{\Omega}$ which truncates on the control region $\Omega$, yields:

Theorem 2.1. For all $\alpha>1 / 2$, the fractional diffusion system:

$$
\partial_{t} \phi+(-\Delta)^{\alpha} \phi=\chi_{\Omega} u, \phi(0)=\phi_{0} \in L^{2}(M), u \in L_{l o c}^{2}\left(\mathbb{R} ; L^{2}(M)\right),
$$

is null-controllable in any time $T>0$. Moreover the controllability cost $C_{T}$ (cf. def.2) over short times satisfies the upper bound:

$$
\forall \beta>1 /(2 \alpha-1), \exists C_{\beta}>0, \exists c_{\beta}>0, \forall T \in(0,1) \quad C_{T} \leqslant C_{\beta} \exp \left(\frac{c_{\beta}}{T^{\beta}}\right) .
$$

REMARK 2.2. This upper bound for the fast controllability cost in the case $\alpha=$ 1 was already stated without proof in [Mil04]. The first motivation for writing the proof came from the preprint version of [MZ04]. The final version of [MZ04] mentions that "a careful analysis of the method of proof in [LR95, LZ98] shows that it works if $\alpha>1 / 2$ " without upper bound.

Micu and Zuazua considered in [MZ04] a similar controllability problems: the space manifold $M$ and the input space $\mathcal{U}$ are one dimensional, $B$ is the multiplication by a shape function $f \in L^{2}(M)$ satisfying extra assumptions (instead of $\chi_{\Omega}$ ). They deduce from the [FR71] a sufficient condition on the Fourier coefficients of $f$ and $\phi_{0}$ (involving $\alpha>1 / 2$ and $T>0$ ) ensuring that there is a $u$ steering $\phi_{0}$ to $O$ in time $T$. Their main negative result is discussed in the next section.

REMARK 2.3. We should comment on the simplest case $\alpha=1$, i.e. diffusion by the heat flow. The fast null-controllability for any control region $\Omega$ has been known for a decade and the fast controllability cost has been investigated, e.g. [FCZ00, Mil04]. It allows us to discuss the optimality of the upper bound in th.2.1. Namely, a lower bound of the same form with equality $\beta=2 /(2-\alpha)$ holds for $\alpha=1$ (cf. [Mil04]). When $M$ is a bounded Euclidean set and $\Delta$ has constant coefficients, [FCZ00] proves that $\lim \sup _{T \rightarrow 0} T \ln C_{T}<\infty$ for any $\Omega$. For general $(M, g)$, but under some geometric condition on $\Omega$, an explicit geometric upper bound on $\lim \sup _{T \rightarrow 0} T \ln C_{T}$ is proved in [Mil04].

2.3. Non controllability of some one dimensional fractional diffusions. Although there are no result for $\alpha \leqslant 1 / 2$ in the setting of the previous section, it seems that the controllability in th.2.1 does not hold for $\alpha \leqslant 1 / 2$ since it does not hold for some similar one dimensional fractional diffusions controllability problems.

Indeed, [MZ04] concerns such a negative result in the setting of "lumped" interior control described in rem.2.2. Micu and Zuazua first recall a result of [Fat66] saying that for any $\alpha \leqslant 1 / 2$ and $T>0$ there is an $f$ and a $\phi_{0}$ that cannot be steered to 0 in time $T$ by any $u$. They improve this result in th.3.1 by describing a large set of non controllable initial states.

The key point of this example compared to the setting of th.2.1 (even when $M$ is one-dimensional) is that the input space $\mathcal{U}$ is one dimensional. This allows to make the well known reduction to some properties of entire functions and exponential sums (cf. [FR71] and references in [Mil04]). Indeed, we point out in the appendix that some abstract systems with finite dimensional inputs satisfying a condition based on Müntz theorem on the completeness of sets of exponentials have a large 
set of non controllable initial states. In particular, it allows us to state here a strong non controllability result for a one dimensional boundary control system (n.b. here the input space is naturally one dimensional without extra assumption on the structure of the source term).

For this result, the manifold is a segment, i.e. $M=(0, L)$. For this result only, we consider the Neumann Laplacian $\Delta_{N}$ which acts as $\Delta$ but has a different domain: $D\left(\Delta_{N}\right)=\left\{\phi \in H^{2}(M) \mid \phi^{\prime}(0)=\phi^{\prime}(L)=0\right\}$. Let $A=-\left(-\Delta_{N}\right)^{\alpha}$ with $\alpha \in(1 / 4,1 / 2]$. Since $\alpha<3 / 4, D(A)$ with the graph norm is $X_{1}=H^{2 \alpha}(0, L)$ (without boundary condition) which injects continuously in the space of continuous functions for $\alpha>1 / 4$. Therefore, $b: \phi \mapsto \phi(L)$ is continuous on $X_{1}$, and thus defines $b$ in the dual $X_{-1}$ of $X_{1}$. N.b. if the metric is not Euclidean, then $\Delta_{N}$ has variable coefficients so that the eigenvalues $\left\{\lambda_{n}\right\}_{n \in \mathbb{N}}$ and eigenfunctions $\left\{\phi_{n}\right\}_{n \in \mathbb{N}}$ are not explicit. But they satisfy $\phi_{n}(L) \neq 0$ and $\lambda_{n} \sim C n^{2 \alpha}$ where $C$ is a negative constant, so that $b_{n}=\left\langle b, \phi_{n}\right\rangle \neq 0$ and property $\left.i i\right)$ of th.A.1 holds for $2 \alpha<1$. Therefore th.A.2 implies:

Theorem 2.4. Assume $b$ is the boundary control operator and $A$ is the fractional Neumann Laplacian defined above with $\alpha \in(1 / 4,1 / 2]$. For all finite linear combination $x^{0} \neq 0$ of the eigenvectors of $A$ and for all $T>0$, there is no input function $u \in L^{2}(0, T ; \mathbb{C})$ such that the solution $x \in C\left(0, T ; X_{-1}\right)$ of $\dot{x}(t)=A x(t)+b u(t)$ with initial state $x(0)=x^{0}$ satisfies $x(T)=0$.

\section{Proof of the MAIN THEOREM}

This section concerns the proof of th.1.1. In a first step, from the stationary condition in def.3, we deduce the observability of low-frequency modes over any positive time in the corresponding dynamics (this is the abstract version of sect.4 in [Mil05]). In a second step, using an abstract version of the iterative control strategy introduced by Lebeau and Robbiano in [LR95] (cf. sect.5 in [Mil05]), we prove the full null-controllability in arbitrarily small time. The main novelty is the last step, in which we estimate the controllability cost as the control time tends to zero.

3.1. From the stationary to the evolution equation. Assume that (3) holds. Let $\tau \in(0,1], \mu \geqslant 1$ and $v_{0} \in \mathbf{1}_{A^{\gamma} \leqslant \mu} \mathcal{H}$. For all $t \in[0, \tau]$, we may apply (3) to $v=e^{-t A} v_{0}$ since it is in $\mathbf{1}_{A^{\gamma} \leqslant \mu} \mathcal{H}$ :

$$
d_{2}^{2} e^{2 d_{1} \mu}\left\|C e^{-t A} v_{0}\right\|^{2} \geqslant\left\|e^{-t A} v_{0}\right\|^{2}=\int_{0}^{\mu} e^{-2 t \lambda^{1 / \gamma}} d\left(E_{\lambda} v_{0}, v_{0}\right) .
$$

First integrating on $[0, \tau]$ with the new variable $s=t / \tau$, then using $\tau \leqslant 1$ and finally $\int_{0}^{1} \exp (-\alpha t) d t=(1-\exp (-\alpha)) / \alpha \geqslant(2 \alpha)^{-1}$ for $\alpha \geqslant \ln 2$ yields:

$$
\begin{aligned}
d_{2}^{2} e^{2 d_{1} \mu} \int_{0}^{\tau}\left\|C e^{-t A} v_{0}\right\|^{2} d t \geqslant & \tau \int_{0}^{1} \int_{0}^{\mu} e^{-2 \tau s \lambda^{1 / \gamma}} d\left(E_{\lambda} v_{0}, v_{0}\right) d s \\
& \geqslant \tau \int_{0}^{1} e^{-2 s \mu^{1 / \gamma}} d s \int_{0}^{\mu} d\left(E_{\lambda} v_{0}, v_{0}\right) \geqslant \frac{\tau}{4 \mu^{1 / \gamma}}\left\|v_{0}\right\|^{2} .
\end{aligned}
$$

Therefore, for any $D_{1}>d_{1}$, there is a $D_{2}>0$ such that fast observability at low-frequencies for $e^{-t A}$ holds: there are positive constants $D_{1}$ and $D_{2}$ such that

$$
\forall \mu>1, \forall \tau \in(0,1], \forall v_{0} \in \mathbf{1}_{A^{\gamma} \leqslant \mu} \mathcal{H}, \quad\left\|e^{-\tau A} v_{0}\right\| \leqslant \frac{D_{2}}{\sqrt{\tau}} e^{D_{1} \mu}\left\|C e^{-t A} v_{0}\right\|_{L^{2}(0, \tau ; \mathcal{U})} .
$$

By duality (cf. [DR77]), this is equivalent to the following null-controllability: for all $\tau \in(0,1]$ and $\mu>1$, there is a bounded operator $S_{\mu}^{\tau}: \mathcal{H} \rightarrow L^{2}(0, \tau ; \mathcal{U})$ 
such that for all $\phi_{0} \in \mathbf{1}_{A^{\gamma} \leqslant \mu} \mathcal{H}$, the solution $\phi \in C([0, \infty), \mathcal{H})$ of (1) with control function $u=S_{\mu}^{\tau} \phi_{0}$ satisfies $\mathbf{1}_{A^{\gamma} \leqslant \mu} \phi=0$ at $t=\tau$. Moreover, we have the cost estimate: $\left\|S_{\mu}^{\tau}\right\| \leqslant \frac{D_{2}}{\sqrt{\tau}} e^{D_{1} \mu}$.

3.2. From low-frequencies to full controllability. Assume that (3) holds with $\gamma \in(0,1)$. We introduce a dyadic scale of frequencies $\mu_{k}=2^{k}(k \in \mathbb{N})$ and a sequence of time intervals $\tau_{k}=\sigma_{\delta} T / \mu_{k}^{\delta}$ where $\delta \in\left(0, \gamma^{-1}-1\right)$ and $\sigma_{\delta}=$ $\left(2 \sum_{k \in \mathbb{N}} 2^{-k \delta}\right)^{-1}>0$, so that the sequence of times defined recursively by $T_{0}=0$ and $T_{k+1}=T_{k}+2 \tau_{k}$ converges to $T$. The strategy of Lebeau and Robbiano in [LR95] is to steer the initial state $\phi_{0}$ to 0 through the sequence of states $\phi_{k}=$ $\phi\left(T_{k}\right) \in \mathbf{1}_{A^{\gamma}>\mu_{k-1}} \mathcal{H}$ at frequencies converging to infinity by applying recursively the control function $u_{k}=S_{\mu_{k}}^{\tau_{k}} \phi_{k}$ to $\phi_{k}$ during a time $\tau_{k}$ and zero control during a time $\tau_{k}$. This strategy is successful if $\phi_{k}$ tends to zero and the control function $u(t)=\sum_{k} \mathbf{1}_{0 \leqslant t-T_{k} \leqslant \tau_{k}} u_{k}(t)$ is in $L^{2}(0, T ; \mathcal{H})$. Since the cost estimate above implies $\left\|S_{\mu_{k}}^{\tau_{k}}\right\| \leqslant D_{2} e^{D_{1} \mu_{k}} / \sqrt{\tau_{k}}$, it only remains to check that:

(5) $\quad \varepsilon_{k}=\left\|\phi_{k}\right\|$ and $C_{k}=D_{2} e^{D_{1} \mu_{k}} / \mu_{k}^{\delta / 2}$ satisfy $\lim _{k} \varepsilon_{k}=0$ and $\sum_{k \in \mathbb{N}} C_{k}^{2} \varepsilon_{k}^{2}<\infty$.

Since $\mathbf{1}_{A^{\gamma} \leqslant \mu_{k}} \phi\left(T_{k}+\tau_{k}\right)=0$, we have $\varepsilon_{k+1} \leqslant e^{-\tau_{k} \mu_{k}^{1 / \gamma}}\left\|\phi\left(T_{k}+\tau_{k}\right)\right\|$. The expression of $\phi\left(T_{k}+\tau_{k}\right)$ in terms of the source term $u_{k}$ (Duhamel's formula) and $\left\|e^{t \Delta}\right\| \leqslant 1$ (contractivity of the heat semigroup) imply $\left\|\phi\left(T_{k}+\tau_{k}\right)\right\| \leqslant 2\left(\varepsilon_{k}+\sqrt{\tau_{k}}\left\|u_{k}\right\|\right)$. Therefore $\varepsilon_{k+1} \leqslant 2 e^{-\tau_{k} \mu_{k}^{1 / \gamma}}\left(1+\sqrt{\tau_{k}} C_{k}\right) \varepsilon_{k}$. Since $C_{k+1} / C_{k}=e^{D_{1} \mu_{k}} / 2^{\delta / 2}$, we deduce:

$$
\frac{C_{k+1} \varepsilon_{k+1}}{C_{k} \varepsilon_{k}} \leqslant 2 e^{-\tau_{k} \mu_{k}^{1 / \gamma}}\left(1+D_{2} e^{D_{1} \mu_{k}}\right) e^{D_{1} \mu_{k}} / 2^{\delta / 2} \leqslant D_{3} \exp \left(2 D_{1} \mu_{k}-\sigma_{\delta} T \mu_{k}^{\gamma^{-1}-\delta}\right),
$$

for some $D_{3}>0$. Since $\gamma^{-1}-\delta>1$ this implies $\sum_{k \in \mathbb{N}} C_{k}^{2} \varepsilon_{k}^{2}<\infty$, which proves (5) and completes the proof of the first assertion of th.1.1.

3.3. Estimate of the controllability cost over short times. To estimate the cost $C_{T} \leqslant\left(\sum_{k \in \mathbb{N}} C_{k}^{2} \varepsilon_{k}^{2}\right)^{1 / 2} / \varepsilon_{0}$ as $T \rightarrow 0$, we define $\rho_{k}$ which satisfies:

$$
\exists D_{4}>0, \quad \rho_{k}:=\left(\frac{C_{k+1} \varepsilon_{k+1}}{C_{k} \varepsilon_{k}}\right)^{2} \leqslant D_{4} \exp \left(4 D_{1} \mu_{k}-2 \sigma_{\delta} T \mu_{k}^{\gamma^{-1}-\delta}\right)
$$

according to the last estimate of the previous subsection. Since

$$
\sum_{0 \leqslant k \leqslant l-1} \mu_{k}^{\gamma^{-1}-\delta}=\frac{2^{\left(\gamma^{-1}-\delta\right) l}-1}{2^{\left(\gamma^{-1}-\delta\right)}-1} \geqslant \mu_{l}^{\gamma^{-1}-\delta} \frac{1-1 / 2}{2^{\left(\gamma^{-1}-\delta\right)}}=\mu_{l-1}^{\gamma^{-1}-\delta} / 2
$$

we have, with $q:=2^{\gamma^{-1}-\delta} \in\left(2,2^{\gamma^{-1}}\right)$ and $T^{\prime}:=\sigma_{\delta} T / q$ :

$$
\prod_{0 \leqslant k \leqslant l-1} \rho_{k} \leqslant D_{4}^{l} \exp \left(4 D_{1} \mu_{l}-2 \sigma_{\delta} T \mu_{l-1}^{\gamma^{-1}-\delta}\right)=D_{4}^{l} \exp \left(4 D_{1} 2^{l}-T^{\prime} q^{l}\right) \text {. }
$$

Therefore the cost satisfies for some $D_{5}>0$ :

$$
\sum_{k \geqslant 0} C_{k}^{2} \varepsilon_{k}^{2}=C_{0}^{2} \varepsilon_{0}^{2}\left(1+\sum_{l \geqslant 1} \prod_{0 \leqslant k \leqslant l-1} \rho_{k}\right) \leqslant \varepsilon_{0}^{2} D_{5}\left(1+\sum_{k \geqslant 1} \exp \left(4 D_{1} 2^{k}-T^{\prime} q^{k}\right)\right) .
$$

To estimate the last sum, we shall use the simple estimate:

$$
f(t):=\sum_{k \geqslant 1} \exp \left(-t q^{k}\right) \leqslant \sum_{k \geqslant 1} \exp (-t k) \sim_{t \rightarrow 0} \frac{1}{t} .
$$


Optimizing the function $h(x):=4 D_{1} 2^{x}-T^{\prime} q^{x}$ yields that, for $\varepsilon \in(0, \ln 2 / \ln q)$ : $h(x) \leqslant h\left(x_{0}\right)-(1-\varepsilon) T^{\prime} q^{x}$ for $x \geqslant 0$, with $x_{0}:=\ln \left(4 D_{1} \ln 2 /\left(T^{\prime} \ln q\right)\right) / \ln (q / 2)$. Therefore, using $x_{0} \sim_{T^{\prime} \rightarrow 0} \ln \left(1 / T^{\prime}\right) / \ln (q / 2)$, with $\beta_{q}:=\left(\frac{\ln q}{\ln 2}-1\right)^{-1}$ :

$$
\begin{aligned}
& \sum_{k \geqslant 1} \exp \left(4 D_{1} 2^{k}-T^{\prime} q^{k}\right) \leqslant \exp \left(h\left(x_{0}\right)\right) f\left((1-\varepsilon) T^{\prime}\right) \\
= & A q^{x_{0}} \frac{1}{\beta_{q}} f\left((1-\varepsilon) T^{\prime}\right) \sim_{T^{\prime} \rightarrow 0} \frac{1}{T^{\prime \beta_{q}}} \frac{1}{\beta_{q}} f\left((1-\varepsilon) T^{\prime}\right) .
\end{aligned}
$$

Thanks to (6), this yields the cost estimate:

$$
\exists D_{6}>0, \exists D_{7}>0, \quad \sum_{k \geqslant 0} C_{k}^{2} \varepsilon_{k}^{2} \leqslant \varepsilon_{0}^{2} D_{6} \exp \left(\frac{D_{7}}{T^{\prime \beta_{q}}}\right)
$$

Since $T^{\prime}=\sigma_{\delta} T / q$ and, as $\delta$ increases to $\gamma^{-1}-1, q=2^{\gamma^{-1}-\delta}$ increases to $2^{\gamma^{-1}}$ and $\beta_{q}$ decreases to $\gamma /(1-\gamma)$, the second assertion of th.1.1 is proved.

\section{Appendix A. LACK Of CONTROLlability BASEd ON MÜNtZ TheOrem}

This appendix concerns controllability systems having a Riesz basis of eigenvectors and a one-dimensional input space. It is well-known that their exact, null and approximate controllability are related to properties of sets of exponentials (cf. [AI95]). Such systems where recently considered in [RW00], [JZ01] and [JP04]. In particular a necessary and sufficient condition for null-controllability in terms of the eigenvalues is given in [JP04]. This condition is enough to prove that nullcontrollability does not hold in sect.2.3. Here, we give a sufficient condition in terms of the eigenvalues for a much stronger property : finite linear combination of the eigenvectors are initial state which cannot be steered to zero by any input function.

The generalized Müntz theorem referred to in the title of this appendix is the following th. 7 of [Red77] (the original Müntz-Szász theorem concerned the approximation by power functions $x \mapsto x^{\lambda_{n}}$, with positive exponents $\lambda_{n}$, instead of exponentials; we refer to[BE96] for more results and references):

Theorem A.1. Let $\left\{\lambda_{n}\right\}_{n \in \mathbb{N}}$ be a sequence of distinct non zero complex numbers and let $\left\{e_{n}\right\}_{n \in \mathbb{N}}$ be the corresponding sequence of exponential functions defined by $e_{n}(t)=\exp \left(\lambda_{n} t\right)$.

If $\left\{\lambda_{n}\right\}_{n \in \mathbb{N}}$ satisfies one of these properties:

i) $\exists \varepsilon>0, \sum_{n} \frac{1}{\left|\lambda_{n}\right|^{1+\varepsilon}}=\infty$,

ii) $\sum_{n}\left|\operatorname{Re} \frac{1}{\lambda_{n}}\right|=\infty$,

iii) $\left\{\lambda_{n}\right\}_{n \in \mathbb{N}}$ increases and there exists a sequence $\left\{\theta_{n}\right\}_{n \in \mathbb{N}}$ of nonnegative real numbers such that $\sum_{n} \frac{1}{n^{\theta_{n}}}<\infty$, and $\sum_{n} \frac{1}{\left|\lambda_{n}\right|^{\theta_{n}}}=\infty$,

then, for all $T>0,\left\{e_{n}\right\}_{n \in \mathbb{N}}$ is complete in $L^{2}(0, T ; \mathbb{C})$, i.e. any function of $L^{2}(0, T ; \mathbb{C})$ is an infinite linear combinations of these exponential functions converging in the norm of this space.

On a Hilbert space $\mathcal{X}$ we consider the system described by the following differential equation for $t \geqslant 0$ :

$$
\dot{x}(t)=A x(t)+b u(t), x(0)=x^{0} \in \mathcal{X}, u \in L_{\mathrm{loc}}^{2}(\mathbb{R} ; \mathbb{C}) .
$$

We assume that $A$ is the infinitesimal generator of a $C_{0}$-semigroup $\left\{e^{t A}\right\}_{t \geqslant 0}$ on $\mathcal{X}$, which has a sequence of normalized eigenvectors $\left\{\phi_{n}\right\}_{n \in \mathbb{N}}$ forming a Riesz basis of 
$\mathcal{X}$, with associated eigenvalues $\left\{\lambda_{n}\right\}_{n \in \mathbb{N}}$, that is, $A \phi_{n}=\lambda_{n} \phi_{n}$. We denote by $\mathcal{X}_{1}$ the Hilbert space obtained by choosing the graph norm on the domain $D(A)$ of the unbounded operator $A$ on $\mathcal{X}$, by $\mathcal{X}_{-1}$ the space dual to $\mathcal{X}_{1}$, and we keep the same notation for the extension of $\left\{e^{t A}\right\}_{t \geqslant 0}$ to a semigroup on $\mathcal{X}_{-1}$. We also assume that the "control vector" $b$ is in $\mathcal{X}_{-1}$ so that the solution $x \in C\left(0, T ; \mathcal{X}_{-1}\right)$ of $(7)$ is defined for $T \geqslant 0$ by the integral formula:

$$
x(T)=e^{T A} x^{0}+\int_{0}^{T} e^{(T-t) A} b u(t) d t .
$$

There is a sequence of eigenvectors $\left\{\psi_{n}\right\}_{n \in \mathbb{N}}$ of $A^{*}$ forming a Riesz basis of $\mathcal{X}$, with associated eigenvalues $\left\{\bar{\lambda}_{n}\right\}_{n \in \mathbb{N}}$, which is bi-orthogonal to $\left\{\phi_{n}\right\}_{n \in \mathbb{N}}$, i.e. $\left\langle\phi_{n}, \psi_{n}\right\rangle=1$ and $\left\langle\phi_{n}, \psi_{m}\right\rangle=0$ if $m \neq n$. We introduce the coefficients $b_{n}=\left\langle b, \psi_{n}\right\rangle$ in the expansion $b=\sum_{n \in \mathbb{N}} b_{n} \phi_{n}$.

Theorem A.2. Assume that $b_{n} \neq 0$ for all $n$ larger than some integer $N_{b}$. If the set of distinct non zero eigenvalues of $A$ satisfies one of the properties stated in theorem A.1, then, for all non zero initial state $x^{0}$ which is a finite linear combination of the eigenvectors $\left\{\phi_{n}\right\}_{n \in \mathbb{N}}$ and for all $T>0$, there is no input function $u \in L^{2}(0, T ; \mathbb{C})$ such that the solution $x \in C\left(0, T ; \mathcal{X}_{-1}\right)$ of $(7)$ satisfies $x(T)=0$.

Proof. Introducing the coefficients $x_{n}(t)=\left\langle x(t), \psi_{n}\right\rangle,(8)$ writes $x_{n}(T)=e^{-\lambda_{n} T} x_{n}^{0}+$ $\int_{0}^{T} e^{-\lambda_{n}(T-t)} b_{n} u(t) d t$. With the notation $e_{n}(t)=\exp \left(\lambda_{n} t\right), x(T)=0$ writes:

$$
\forall n \in \mathbb{N}, \quad-x_{n}^{0}=b_{n} \int_{0}^{T} e_{n}(t) u(t) d t .
$$

We make the assumptions on $\left\{b_{n}\right\}_{n \in \mathbb{N}}$ and $\left\{\lambda_{n}\right\}_{n \in \mathbb{N}}$ of the theorem. Arguing by contradiction, we also assume that there are $T>0, x^{0} \neq 0$ which is a finite linear combination of the $\left\{\phi_{n}\right\}_{n \in \mathbb{N}}$, and $u \in L^{2}(0, T ; \mathbb{C})$ such that $(9)$ holds. Let $x_{N}^{0}$ be the nonzero coefficient of $x^{0}$ with the greatest index, i.e. $x_{N}^{0} \neq 0$ and $x_{n}^{0}=0$ for $n>N$. Let $M=\max \left\{N_{b}, N\right\}$. For all $n>M$, on the one hand $M \geqslant N_{b}$ implies $b_{n} \neq 0$, on the other hand $M \geqslant N$ implies $x_{n}^{0}=0$, so that (9) implies $\int_{0}^{T} e_{n}(t) u(t) d t=0$. The set of distinct non zero values of $\left\{\lambda_{n}\right\}_{n>M}$ also satisfies one of the properties stated in theorem A.1, so that the corresponding subset of $\left\{e_{n}\right\}_{n>M}$ is complete in $L^{2}(0, T ; \mathbb{C})$. In particular, $e_{N}=\sum_{n>M} c_{n} e_{n}$ for some coefficients $\left\{c_{n}\right\}_{n>M} \in l^{2}(\mathbb{C})$. Plugging this expansion in (9) with $n=N$ yields the contradiction:

$$
0 \neq-x_{N}^{0}=b_{N} \sum_{n>M} c_{n} \int_{0}^{T} e_{n}(t) u(t) d t=0 .
$$

REMARK A.3. This abstract theorem applies directly to the context of th.3.1 in [MZ04], since eq.2.10 in [MZ04] corresponds to the hypothesis $b_{n} \neq 0$ for all $n$. In an explicit setting where $\lambda_{n}=n^{2 \alpha}$ with $\alpha \in(0,1 / 2]$, this th.3.1 of Micu and Zuazua describes a much larger set of initial data which cannot be steered to zero.

REMARK A.4. The following weaker result, in the setting of finite-dimensional input space (instead of one-dimensional) but of eigenvectors forming a Hilbert basis (instead of Riesz basis) and of eigenvalues with negative real parts, can be deduced from [AI95] by combining th. III.3.3(d) with th. II.2.4 as in the proof of th. IV.1.3(c): if the eigenvalues violate the Blaschke condition $\sum_{n} \operatorname{Re} \lambda_{n}\left(1+\left|\lambda_{n}\right|^{2}\right)^{-1}>-\infty$, then, for all $T>0$, there is an initial state equal to some eigenvector $\phi_{n}$ which cannot be steered to zero in time $T$ by any input function (n.b. when $\left|\lambda_{n}\right| \rightarrow \infty$, the violation of the Blaschke condition here is equivalent to the property ii) in theorem A.1). 


\section{REFERENCES}

[AI95] S. A. Avdonin and S. A. Ivanov, Families of exponentials, the method of moments in controllability problems for distributed parameter systems, Cambridge University Press, Cambridge, 1995.

[App04] D. Applebaum, Lévy processes - from probability to finance and quantum groups, Notices Amer. Math. Soc. 51 (2004), no. 11, 1336-1347.

[BE96] P. Borwein and T. Erdélyi, The full Müntz theorem in $C[0,1]$ and $L_{1}[0,1]$, J. London Math. Soc. (2) 54 (1996), no. 1, 102-110.

[DR77] S. Dolecki and D. L. Russell, A general theory of observation and control, SIAM J. Control Optimization 15 (1977), no. 2, 185-220.

[Fat66] H. O. Fattorini, Control in finite time of differential equations in Banach space, Comm. Pure Appl. Math. 19 (1966), 17-34.

[FCZ00] E. Fernández-Cara and E. Zuazua, The cost of approximate controllability for heat equations: the linear case, Adv. Differential Equations 5 (2000), no. 4-6, 465-514.

[FR71] H. O. Fattorini and D. L. Russell, Exact controllability theorems for linear parabolic equations in one space dimension, Arch. Rational Mech. Anal. 43 (1971), 272-292.

[GM03] R. Gorenflo and F. Mainardi, Fractional diffusion processes: probability distributions and continuous time random walk, Processes with Long Range Correlations (G. Rangarajan and M. Ding, eds.), Lecture Notes in Physics, vol. 621, 2003, pp. 148-166.

[Han01] A. Hanyga, Multidimensional solutions of space-fractional diffusion equations, R. Soc. Lond. Proc. Ser. A Math. Phys. Eng. Sci. 457 (2001), no. 2016, 2993-3005.

[JL99] D. Jerison and G. Lebeau, Nodal sets of sums of eigenfunctions, Harmonic analysis and partial differential equations (Chicago, IL, 1996), Univ. Chicago Press, Chicago, IL, 1999, pp. 223-239.

[JP04] B. Jacob and J. R. Partington, On controllability of diagonal systems with onedimensional input space, Proc. of the MTNS 2004, Leuven/Belgium, 2004.

[JZ01] B. Jacob and H. Zwart, Exact observability of diagonal systems with a finite-dimensional output operator, Systems Control Lett. 43 (2001), no. 2, 101-109.

[LR95] G. Lebeau and L. Robbiano, Contrôle exact de l'équation de la chaleur, Comm. Partial Differential Equations 20 (1995), no. 1-2, 335-356.

[LZ98] G. Lebeau and E. Zuazua, Null-controllability of a system of linear thermoelasticity, Arch. Rational Mech. Anal. 141 (1998), no. 4, 297-329.

[Mil04] L. Miller, Geometric bounds on the growth rate of null-controllability cost for the heat equation in small time, J. Differential Equations 204 (2004), no. 1, 202-226.

[Mil05] __ Unique continuation estimates for the Laplacian and the heat equation on noncompact manifolds, Math. Res. Lett. 12 (2005), no. 1, 37-47.

[MK04] R. Metzler and J. Klafter, The restaurant at the end of the random walk: recent developments in the description of anomalous transport by fractional dynamics, J. Phys. A 37 (2004), no. 31, R161-R208.

[MZ04] S. Micu and E. Zuazua, On the controllability of a fractional order parabolic equation, to appear in SIAM J. Control Optim., 2004.

[Red77] R. M. Redheffer, Completeness of sets of complex exponentials, Advances in Math. 24 (1977), no. 1, 1-62.

[RW00] R. Rebarber and G. Weiss, Necessary conditions for exact controllability with a finitedimensional input space, Systems Control Lett. 40 (2000), no. 3, 217-227.

[Sat99] K. Sato, Lévy processes and infinitely divisible distributions, Cambridge Studies in Advanced Mathematics, vol. 68, Cambridge University Press, Cambridge, 1999.

[Sei88] T. I. Seidman, How violent are fast controls?, Math. Control Signals Systems 1 (1988), no. 1, 89-95.

[SKB02] I. Sokolov, J. Klafter, and A. Blumen, Fractional kinetics, Physics Today 55 (2002), 48-54.

[SV03] R. Song and Z. Vondraček, Potential theory of subordinate killed Brownian motion in a domain, Probab. Theory Related Fields 125 (2003), no. 4, 578-592.

[Wei89] G. Weiss, Admissible observation operators for linear semigroups, Israel J. Math. 65 (1989), no. 1, 17-43.

Équipe Modal'X, EA 3454, Université Paris X, BÂt. G, 200 Av. De la République, 92001 NANTERRE, FRANCE.

Centre de Mathématiques Laurent Schwartz, UMR CNRS 7640, École Polytechnique, 91128 Palaiseau, France.

E-mail address: miller@math.polytechnique.fr 